\title{
Rotary substates of mitochondrial ATP synthase reveal the basis of flexible $F_{1}-F_{0}$ coupling
}

\author{
B. J. Murphy ${ }^{\dagger, 1}$, N. Klusch ${ }^{\dagger, 1}$, J. Langer ${ }^{2}$, D. J. Mills ${ }^{1}$, Ö. Yildiz ${ }^{1}$, W. Kühlbrandt ${ }^{1}$ \\ ${ }^{1}$ Department of Structural Biology, and ${ }^{2}$ Department of Molecular Membrane Biology, Max Planck Institute of Biophysics, \\ Max-von-Laue Str. 3, Frankfurt 60438, Germany; ${ }^{\dagger}$ These authors contributed equally
}

Abstract:

$F_{1} F_{0}$-ATP synthases play a central role in cellular metabolism, making the energy of the protonmotive force across a membrane available for a large number of energy-consuming processes. We determined the single-particle cryo-EM structure of active dimeric ATP synthase from mitochondria of Polytomella sp. at 2.7- 2.8 ̊ resolution. Separation of 13 well-defined rotary substates by 3D classification provides a detailed picture of the molecular motions that accompany $c$-ring rotation and result in ATP synthesis. Crucially, the $F_{1}$ head rotates along with the central stalk and $c$-ring rotor for the first $\sim 30^{\circ}$ of each $120^{\circ}$ primary rotary step. The joint movement facilitates flexible coupling of the stoichiometrically mismatched $F_{1}$ and $F_{o}$ subcomplexes. Flexibility is mediated primarily by the interdomain hinge of the conserved OSCP subunit, a well-established target of physiologically important inhibitors. Our maps provide atomic detail of the $c$-ring/a-subunit interface in the membrane, where protonation and deprotonation of c-ring cGlu111 drives rotary catalysis. An essential histidine residue in the lumenal proton access channel binds a strong non-peptide density assigned to a metal ion that may facilitate $c$-ring protonation, as its coordination geometry changes with c-ring rotation. We resolve ordered water molecules in the proton access and release channels and at the gating $a \operatorname{Arg} 239$ that is critical in all rotary ATPases. We identify the previously unknown ASA10 subunit and present complete de novo atomic models of subunits ASA1-10, which make up the two interlinked peripheral stalks that stabilize the Polytomella ATP synthase dimer.

\section{One Sentence Summary:}

Mechanisms of proton translocation and flexible $F_{1}-F_{0}$ coupling by rotary substates in a mitochondrial ATP synthase dimer

\section{Main Text:}

Mitochondria carry out controlled oxidation of reduced substrates to generate an electrochemical gradient across the inner mitochondrial membrane. Movement of protons down the gradient through the $\mathrm{F}_{1} \mathrm{~F}_{\mathrm{o}}$-ATP synthase drives the production of the soluble energy carrier ATP by rotary catalysis. The ATP synthases consist of two connected nanomotors: the membrane-embedded $F_{0}$ subcomplex where proton translocation generates torque, and the soluble $F_{1}$ subcomplex where the 
torque powers ADP phosphorylation (for a recent review, see (1)). In $F_{0}$, a ring composed of 8-17 csubunits, each containing a carboxylate ion-binding site, rotates against a bearing formed by a nearhorizontal helix hairpin of the stationary $a$-subunit (2). Two aqueous half-channels at the interface of the $c$-ring and $a$-subunit allow protons to enter and leave the protonation site; upon protonation from the lumenal channel, a c-ring subunit travels by an almost full rotation through the hydrophobic membrane environment before releasing the proton in the matrix channel. In the membrane, the channels are separated by a mere $6 \AA$ (3), with a strictly conserved arginine residue facilitating the passing of deprotonated, but not protonated, $c$-ring subunits. The energy from down-gradient proton translocation powers rotation of the $c$-ring and the firmly attached central stalk (subunits $\gamma, \delta, \varepsilon$ ), which conveys rotation generated in $\mathrm{F}_{\mathrm{o}}$ to power ATP synthesis. A two-helix bundle of the $\gamma$-subunit extends deep into the $\alpha_{3} \beta_{3}$ hexamer of the $F_{1}$ head. Rotation of the central stalk induces conformational changes of subunits $\alpha$ and $\beta$ that result in ADP phosphorylation. The two-domain oligomycin-sensitivity conferring protein, $O S C P$, connects the $F_{1}$ head to a membrane-anchored peripheral stalk stator, preventing unproductive rotation of $F_{1}$.

The dimeric mitochondrial ATP synthase from the chlorophyll-less unicellular alga Polytomella sp. contains the signature ATP synthase subunits $\alpha_{3}, \beta_{3}, \gamma, \delta, \varepsilon, c_{10}$, $a$, and $O S C P$, while peripheral stalk and other membrane protein subunits typical of mammalian and fungal ATP synthases (1) are replaced in Polytomella and related species by proteins known as ATP-synthase associated (ASA) proteins, which bear no homology to other known ATP synthase components $(4,5)$. These subunits form a bulky peripheral stalk that links the two ATP synthase complexes into a stable dimer.

ATP synthase must couple translocation of eight to seventeen protons (1), depending on c-ring size, with phosphorylation of three molecules of ADP in the catalytic $\beta$-subunit sites of the $\mathrm{F}_{1}$ head. The stoichiometric mismatch of $F_{1}$ and $F_{0}$ poses a challenge to efficient energy conversion in the complex $(6,7)$. It has been proposed that the central stalk mediates flexible coupling between $F_{1}$ and $F_{0}(8,9)$. Some authors suggest energy is stored by coiling of the $\gamma$-subunit two-helix bundle (10); others propose that energy is stored at the 'foot', where $\gamma$ and $\varepsilon$ are attached to the $c$-ring (9). Recent cryoEM studies resolving rotary states of mitochondrial (11), bacterial (12) and chloroplast (13) ATP synthase have all found that the central stalk rotates as a rigid body. Substantial flexibility in the peripheral stalk is observed between rotary states (11-14), which suggests that peripheral stalk bending contributes to elastic energy storage. The robust, rigid peripheral stalks of the Polytomella ATP synthase make it an ideal system for examining the dynamics revealed by rotary states and substates by high-resolution cryo-EM, and for testing the idea that peripheral stalk flexibility is required for ATP synthase function. 


\section{High-resolution map of a complete F-type ATP synthase dimer}

Cryo-EM single-particle analysis of purified and active (Figure S1) Polytomella ATP synthase dimer yielded a map at $2.94 \AA ̊$ overall resolution (Table S1). Local masking improved the resolution to 2.7$2.8 \AA$ in overlapping regions covering the full complex (Figure S1, S2, S3; Table S2, Movie S1). Symmetry expansion allowed each monomer to be treated independently in image analysis, and 3D classification of the central stalk and $F_{1}$ head enabled separation of three primary rotary states, at resolutions of 2.8-2.9 $\AA$ in the $\mathrm{F}_{1}$ head and rotor (Figure 1). The nucleotide-binding sites $\beta_{T P}$ and $\beta_{D P}$ contain $\mathrm{Mg}^{2+} \mathrm{ADP}$ (Figure $1 \mathrm{~b}, \mathrm{c}, \mathrm{d}$ ) and $\beta_{E}$ is empty; this is the ADP-bound complex, which is to be expected as it was prepared under substrate-depleted conditions. The 'arginine finger' (15) of $\alpha \operatorname{Arg} 429$ extends toward the nucleotide phosphate in $\beta_{\mathrm{DP}}$ but away from it in $\beta_{\mathrm{TP}}$ (Figure 1c,d). Our map provides the highest available resolution of a complete ATP synthase. Cryo-EM data were complemented by genomic sequencing and mass spectrometry analysis of the purified protein, enabling us to build a full atomic model of the $1.58 \mathrm{MDa}$ complex, with 62 copies of 18 different subunits and a total of 14,240 residues fitted. (Figure 1; Figure S4; Table S3). The model includes the completed polypeptide sequence of subunits $A S A 2$ and $\varepsilon$ and the previously unknown subunit $A S A 10$.

\section{Rotary substates reveal concerted rotation of $F_{1}$ and central stalk}

To describe the broad range of thermally-accessible motion of $F_{1} F_{0}$-ATP synthases, we sorted the symmetry-expanded dataset into 12 classes. Further subdivision of a mixed class yielded a total of 13 unique rotary substates (Figure S2) which were refined to 2.8-4.2Å resolution (Figure S3; Table S2). The subclasses can be placed into a meaningful sequence according to the rotational position of the rotor with respect to the stator. Each subclass was assigned to one of three primary rotary states (Figure S1), with six subclasses for state 1 (1A-1F) (Figure 2), four for state 2 (2A-2D) and three for state $3(3 \mathrm{~A}-3 \mathrm{C})$. The 3 primary states are separated from one another by $\sim 120^{\circ}$ rotation of the central stalk within the $F_{1}$ head (Figure $1 a$, inset; Figure S2); in contrast, substates of any given rotary state differ by concerted rotation of the $F_{1}$ head and rotor within the first $15^{\circ}$ to $32^{\circ}$ of each $\sim 120^{\circ}$ step (Figure 2; Figure 3; Movie S2, S3). Contact between the $\beta_{E}$ site and the $\gamma$-subunit (the ' $\beta$-catch loop' (16)) is maintained between substates (Figure S5; Movie S2), as is the nucleotide occupancy of $\beta$ subunits (Figure $3 \mathrm{~b}$ ). The pivot point of this movement is the single peptide chain connecting the two OSCP domains (henceforth the 'OSCP hinge') (Figure 4; Movie S4), while the top of the peripheral stalk flexes only slightly. In comparing all 13 substates, no coiling of the central stalk is apparent, but the extreme C-terminus of $\gamma$ bends slightly in some substates.

It is clear that in the first $\sim 30^{\circ}$ of rotary steps 1 and 2 , the $c$-ring advances by almost one subunit with respect to the $a$-subunit in moving between substates (Figure 3a), while the position of $\gamma$ with 
respect to the $\alpha_{3} \beta_{3}$ hexamer remains virtually unchanged (Figure 3b; Figure $\mathrm{S} 5$ ). Between substates of state 3 , the $c$-ring advances by about half a subunit (Figure $3 a$ ). As the rotor moves a further $\sim 90^{\circ}$ to the starting position of the next primary rotary state (e.g. from state $1 \mathrm{~F}$ to $2 \mathrm{~A}$ ), the $\mathrm{F}_{1}$ head recoils by $\sim 30^{\circ}$ to its original position, for a cumulative $\sim 120^{\circ}$ power stroke of $\gamma$ within $\mathrm{F}_{1}$ for this step. Given that this motion is thermally accessible for the complex at equilibrium, it would almost certainly contribute to flexible coupling of $F_{1}$ and $F_{0}$ also under turnover conditions.

The interaction between $F_{1}$ and $\gamma$ appears strongest in the catch loop region (16) of $\beta_{E}$, where conserved residues $\beta$ Asp318, $\beta$ Asp321, and $\beta$ Thr320 ( $\beta$ Asp302, $\beta$ Asp305, $\beta$ Thr304 in E. coli) form ionic and hydrogen-bond interactions with $\gamma \operatorname{Arg} 296$ and $\gamma \mathrm{G} \ln 297$ (EC $\gamma \operatorname{Arg} 268$ and $\gamma \mathrm{G} \ln 269$ ). The salt bridge between $\beta$ Asp321 and $\gamma \operatorname{Arg} 296$ forms via a resolved water molecule (Figure S5). Throughout the $20^{\circ}-30^{\circ}$ concerted rotation of $F_{1}$ with $\gamma$, the interaction between the $\beta_{E}$ catch loop and $\gamma$ is maintained, although subtle loop movements suggest that the hydrogen-bonding partner of $\beta$ Asp318 changes from $\gamma \mathrm{G} \ln 297$ to $\gamma$ Asn293 between substates (e.g. from state $1 \mathrm{~A}$ to $1 \mathrm{~F}$; Figure S5).

Disruption of these interactions by mutation in E. coli eliminated or strongly reduced ATP hydrolysis activity and the ability to grow on succinate (16). A proposed function of such an interaction is that, in ATP synthesis mode, it would stall rotation of $\gamma$ until the $\beta_{E}$ site has bound ADP, to prevent unproductive rotation (16), although the dramatic impact of catch loop mutations on both synthesis and hydrolysis activity implies that this region plays a broader role in the conformational changes required for catalysis. Our findings make it clear that this interaction does not stall rotation completely; rather, a proton may be translocated at $F_{0}$ while the $F_{1}$ head waits for substrate binding. Studies aiming to characterize flexible coupling have focused on the central stalk (17), often working with an $F_{1}$ and rotor subcomplex. When the whole $F_{1} F_{0}$ complex was examined, it was nonetheless normally attached to substrate and reporter molecules at subunits $\beta$ and $c$ to probe for flexibility along the $F_{1}-F_{0}$ axis $(9,18,19)$. A recent single-molecule study of $F_{1} F_{0}$ reported forward and backward stepping of the rotor by $\sim 30^{\circ}$, i.e. up to one $c$-subunit (18). However, with $F_{1}$ anchored to the support by subunit $\beta$ and a probe attached to $c$, flexibility at $O S C P$ would not be detected. The observed $\sim 30^{\circ}$ stepping angle appears to reflect regular energy minima in the $c$-ring/ $a$-subunit interaction rather than joint rotation of the $c$-ring and central stalk with $F_{1}$. Single-molecule studies of flexible coupling may need to explore a wider range of anchoring points.

Our structure indicates that bending at the OSCP hinge (Figure 4; Movie S4), by mediating flexible coupling between $F_{1}$ and $F_{0}$, plays an important mechanistic role in ATP synthesis. A body of literature suggests that this process has broad physiological implications, with a number of regulatory mechanisms acting on $O S C P$. The regulator of mitochondrial permeability transition, 
cyclophilin D, binds to OSCP and inhibits ATP synthase activity (20), as does the immunomodulator Bz-423, which affects both $V_{\max }$ and $K_{M}$ for ATP hydrolysis (21). Acetylation of a conserved lysine near the hinge region of OSCP in response to exercise stress affects mitochondrial ATP levels (22). The hormone estrogen binds to $\operatorname{OSCP}(23)$ and inhibits ATP synthase activity $(24,25)$ with possible relevance to neuroprotection (26). The tumor suppressor p53, under non-stress conditions, binds to OSCP and promotes increased $\mathrm{O}_{2}$ consumption and decreased ROS levels in mitochondria (27). These diverse and potent modulations of ATP synthase activity cannot be reconciled with a model of ATP synthase in which OSCP forms a static bridge between the $F_{1}$ head and peripheral stalk. Our results show that, on the contrary, OSCP plays a dynamic role in the flexible coupling of $F_{0}$ and $F_{1}$. The concept of a dynamic OSCP will be essential to understand and exploit modulation of ATP synthase activity, with relevance to neurodegeneration and traumatic brain injury, ischemia-reperfusion injury, and immunomodulation, among others.

Several lines of evidence support the idea that the mechanism of flexible coupling is conserved across all types of rotary ATP synthases: 1 ) the structure and polypeptide sequences of $\beta, \gamma$, and OSCP subunits involved in this process are highly conserved; 2 ) a canonical OSCP (or $\delta$ in chloroplasts and bacteria) is found in all three lineages of ATP synthase known to have independently acquired novel peripheral stalk components $(28,29) ; 3)$ a cryo-EM study of bovine ATP synthase (11) identified rotary substates of this complex, albeit at lower resolution, indicating rotation of $F_{1}$ relative to the remainder of the complex. Re-evaluating these results in light of our data, they do in fact show concerted rotation of $F_{1}$ with the central stalk; 4 ) well-known inhibitors and regulators of ATP synthase bind to $O S C P$, affecting the catalytic rate and $\mathrm{K}_{M}(21,30)$, suggesting a mechanistic rather than merely structural role for this subunit.

\section{The essential His-248 ligates a metal ion that is sensitive to $c$-ring rotation}

In respiring mitochondria, the $\mathrm{pH}$ of the lumen is $7.2(31)$, while the $\mathrm{pK}_{\mathrm{a}}$ of the $c$-ring carboxylate in an aqueous environment is around 4.5 (32); thus, protonation of the $c$-ring needs to be assisted by the local environment in the lumenal entrance channel in order to achieve high turnover rates. Mutational studies in E. coli have established that the well-conserved lumenal channel environment is necessary for activity, with residues aGlu219 and $a$ His245 essential for both ATP synthesis and proton permeability of $\mathrm{F}_{\mathrm{o}}$ in membranes stripped of $\mathrm{F}_{1}$. The mutations $a \mathrm{E} 219 \mathrm{H}$ and $a \mathrm{H} 245 \mathrm{E}$ show low levels of activity, while the double mutant is more active than either single mutant (33), suggesting that the residues interact directly to facilitate $c$-ring protonation. Our map shows a strong nonpeptide density ligated by aHis248 (aGlu219 in E.coli) and $a \mathrm{His} 252$ of $a \mathrm{H} 5$ (Figure 5) with bond lengths of $2.1-2.2 \AA$. The strong density and coordination environment favor assignment to a metal ion, provisionally annotated as $\mathrm{Zn}^{2+}$, on account of its abundance, flexible coordination geometry and 
lack of redox activity. At high threshold levels the bound species appears diatomic, perhaps due to a heavy ligand (Figure $5 b, f-h$ ). Both ligating His residues are present in mammalian ATP synthases (Figure S6). The $a$ His248 position is occupied by His or Glu in all F-type ATP synthases, with the notable exception of $\mathrm{Na}^{+}$-translocating complexes (Figure S6). Given its likely role in protonating the $c$-ring, this site thus appears to contribute to selectivity for $\mathrm{H}^{+} \mathrm{vs} . \mathrm{Na}^{+}$, in addition to known differences in the $c$-ring binding site (34). Recently published high-resolution cryo-EM maps of yeast mitochondrial (35) and spinach chloroplast ATP synthase (13) both indicate a strong, unattributed non-peptide density at the same position in the lumenal channel, near the residue equivalent to aHis248 (aHis185 and aGlu198, respectively) (Figure S6). In both instances, a single strong density was observed as compared to the two densities in our map, which may reflect the lower local resolution (3.6 ̊̊ and $3.4 \AA$ ) of the yeast and spinach maps.

Masked 3D classification of the $c$-ring and $a$-subunit yields two distinct $c$-ring rotary positions at $2.7 \AA$ and $3.1 \AA$ A resolution, representing 58\% and 33\% of particles (Figure S2). The positions differ by rotation of the $c$-ring by approximately one third of a $c$-ring subunit $\left(13^{\circ}\right)$ (Figure $\left.5 f\right)$. Comparing these maps, it is clear that the configuration of the metal ion in the lumenal channel changes with rotation of the $c$-ring, with the metal-ligand distance measuring 2.3 and $3.7 \AA$ for $c$-ring positions 1 and 2 (Figure 5f-h). Sidechains of aGlu172 and ASA6GIn93 coordinate the ligand in state 2, with approximate distances of 2.8 and $2.2 \AA$. We observe a slight but significant change in curvature of hairpin helix $\mathrm{H} 5$ between the two c-ring positions (Figure $5 f$ ), which is likely due to mechanical pressure of the $c$-ring subunit against the helix, with flexibility of $\mathrm{H} 5$ facilitated by the conserved aGly247 residue. The simplest explanation of our observations is therefore that the rotating $c$-ring pushes on $a \mathrm{H} 5$, inducing a slight change in curvature, which in turn changes the coordination of the bound metal ion. In both positions, distances between the Hise $\mathrm{N}$ and metal ion are in the 2.1-2.2 $\AA$ range, consistent with dative bonds and thus with unprotonated HiseN. Available structural and mutational data strongly suggest that the role of $a \mathrm{His} 248$ is to protonate $a \mathrm{Glu} 288$, which would require that $a \mathrm{His} 248$ is itself transiently protonated. Protonation of either His or Glu at this position would preclude their ability to coordinate a metal ion. This suggests the existence of a third state in which $a \mathrm{His} 248$ is protonated, leaves the metal ion and approaches $a \mathrm{Glu} 288$ more closely than the distance $a \mathrm{His} 248 \mathrm{~N} \varepsilon-a \mathrm{Glu} 2880$ of $5.5 \AA$ in our model, which is too far for direct proton transfer. If protonation of $a \mathrm{Glu} 288$ by $a \mathrm{His} 248$ is very fast, the structure of this state would not be resolved.

Although further work will be needed to clarify the identity, dynamics, and function of this metal ion, the following working model may help to direct future studies: physical pressure of the $c$-ring on $a \mathrm{H} 5$ stretches the $a$ His248-metal ion bond, favoring its protonation. Upon protonation aHis248 leaves the metal ion and transfers a proton to $a \mathrm{Glu} 288$, which is then passed on to the $c$-ring glutamate, most 
likely via a water, since the residues do not appear to be able to make direct contact at any rotary position of the $c$-ring. Such a mechanism would synchronize proton delivery with c-ring rotation.

\section{Ordered water in the aqueous half-channels and at the essential Arg-239}

The membrane-embedded $a$-subunit and $c$-ring together define the pathway for protons to move across the inner mitochondrial membrane (Figure 5a). The sequences of both subunits are very similar to those of other ATP synthases, even those of bacteria and chloroplasts (1). Previously we showed (3) that the aqueous half-channels that conduct protons to and away from the $c$-ring glutamate are separated by a distance of 5-7 $\AA$ in the centre of the membrane. We now present a high-resolution structure of this critical region. Our map shows ordered water molecules within both channels (Figure $5 c-e$ ). In the matrix channel, a water molecule is coordinated by cSer112 opposite the ion-binding cGlu111 of the adjacent $c$-subunit, favoring the idea that the $c$-ring glutamate may be directly deprotonated by water in this location. An interesting finding is the density of two water molecules enclosed by the strictly conserved $a \operatorname{Arg} 239$ and $a \mathrm{G} \ln 295$, in a pocket which appears not to be continuous with the aqueous channels (Figure 5e). The interaction of these highly conserved residues with each other via a water molecule would stiffen the $\operatorname{aArg} 239$ sidechain. In the lumenal proton access channel the well-ordered water lies between the assigned metal ion density and the strictly conserved $a \operatorname{Arg} 239$. Examining these waters, it is clear that the channel extends to the $a / c$ interface close to $\operatorname{aArg} 239$, as previously reported (3). Although the $c$-ring glutamate is exposed to the aqueous environment immediately adjacent to $\operatorname{aArg} 239$, the results of mutational studies of $a$ His 245 and $a$ Glu219 in E. coli suggest that direct protonation of the $c$-ring from water in the channel is rare; rather, the c-ring is likely protonated as it rotates past $a \mathrm{Glu} 288$ (aHis245 in E. coli), immediately before passing into the hydrophobic lipid bilayer environment. It is therefore worthwhile to consider the role of water in the lumenal channel permeating all the way to $\operatorname{aArg} 239$. Two explanations seem possible: 1 ) the aqueous environment primes the $c$-ring glutamate for protonation by inducing an outward-facing conformation (32) and/or 2) the small distance between half-channels, which would generate a substantial field and resulting torque on the $c$-ring glutamate, is needed to overcome the energetic barrier of moving the $c$-ring glutamate past the gating Arginine $239(1)(3)$.

\section{Novel C-terminal $\beta$-extensions contact the adjacent $\alpha$-subunit}

In chlorophycean algae that include Polytomella, the mitochondrial catalytic $\beta$-subunit resembles those of the canonical ATP synthases closely, except that the chlorophycean subunit has acquired a 60-residue C-terminal extension. Our map shows that this extension wraps vertically around the adjacent $\alpha$-subunit (Movie S5) and moves with subunit $\beta$ as it adopts an open or closed conformation. A $\sim 15$-residue $\mathrm{N}$-terminal extension of the $\alpha$-subunits unique to chlorophycean algae 
is in a different configuration for each subunit (Figure 4b, Movie S4). One of these extensions, together with the distal OSCP domain, anchors one $\alpha$-subunit to the peripheral stalk, while the other two form short helix bundles with the proximal globular domain of $O S C P$, attaching it to $F_{1}$. The $\mathrm{N}$ and C-terminal extensions would enhance the stability of the chlorophycean mitochondrial ATP synthase, which may be an advantage under stress conditions, as when Polytomella converts to a dormant cyst in nutrient-depleted environments (36).

\section{ATP-synthase associated proteins 1-10 form a rigid peripheral stalk}

In Polytomella, dimer formation is mediated by the peripheral stalk consisting of ten ASA subunits that have no homologues in other known ATP synthases outside this class of green algae. We have modelled all nine previously described ASA subunits unambiguously into the $2.7 \AA$ map (Figure 1; Figure S4; Movie S1), as well as an unknown subunit that we term ASA10 (Figure S7). Mass spectrometry of the purified complex and DNA sequencing of the $\sim 50 \mathrm{Mb}$ Polytomella genome allowed us to identify ASA10 (Figure S7) and to obtain complete sequences for subunits $\varepsilon$ and $A S A 2$ (Figure S8). All subunits were built into the cryo-EM map de novo, as there are no homologous structures in the database.

The stability of the Polytomella dimer is due to the rigid peripheral stalks and their close interaction. ASA1 is at the core of the soluble stalk region, and forms a bridge between monomers with a helixturn-helix motif $75 \AA ̊$ above the membrane surface (Figure S9). $F_{0}$ has six trans-membrane helices and four long, membrane-intrinsic helices which form the two helix hairpins of the conserved $a$-subunit (2). Of the six trans-membrane helices, two belong to $A S A 6$ and one each to $A S A 5, A S A 8, A S A 9$, and $A S A 10$. The two $A S A 10$ subunits form the primary dimer interface on the lumenal side of the membrane, and their C-termini are intertwined (Figure S7, S9). Little or no other direct interaction is seen within the membrane, with lipids or detergent filling the space between monomers. ASA3 forms the characteristic Armadillo repeat domain above the membrane surface on the matrix side, and $A S A 2, A S A 4$ and $A S A 7$ sit atop the peripheral stalk.

\section{Outlook}

We have determined the structures of Polytomella ATP synthase in 13 rotary substates, allowing us to visualize most if not all of its thermally-accessible conformations. Critically, we have shown that the $F_{1}$ head and rotor move together through a $20^{\circ}-30^{\circ}$ rotation; thus, the $c$-ring rotates relative to the $a$-subunit while the position of the central stalk within the $F_{1}$ head is preserved (Figure 6). This movement ensures flexible coupling of the two symmetry-mismatched nanomotors of ATP synthase, $F_{o}$ and $F_{1}$, for all possible $c$-ring stoichiometries. Flexible coupling appears to be mediated predominantly by $O S C P$, which is emerging as an important target of cellular and pharmacological 
control. Whereas most previous structural studies of ATP synthase have characterized inhibited complexes, this work has instead examined the product-bound form of the complex, not autoinhibited and free of any added inhibitors, which may account for the increased conformational flexibility we observe. Further studies will address the energetics of OSCP bending, and integrate these results into a full catalytic scheme of ATP synthesis. A metal ion bound at the conserved residue $a \mathrm{His} 248$ within the lumenal channel is sensitive to the $c$-ring rotary state and is likely to play an important role in protonating the $c$-ring carboxylate. An important unanswered question is how the strictly conserved $a \operatorname{Arg} 239$ facilitates movement of deprotonated, but not protonated, $c$-ring subunits between the aqueous half-channels. The high-resolution detail provided by our model will be important for answering this question.

\section{References and Notes:}

1. W. Kühlbrandt, Structure and mechanisms of F-type ATP synthases. Annu Rev Biochem (2019).

2. M. Allegretti et al., Horizontal membrane-intrinsic $\alpha$-helices in the stator a-subunit of an F-type ATP synthase. Nature. 521, 237-240 (2015).

3. N. Klusch, B. J. Murphy, D. J. Mills, Ö. Yildiz, W. Kühlbrandt, Structural basis of proton translocation and force generation in mitochondrial ATP synthase. elife. 6 (2017), doi:10.7554/eLife.33274.

4. M. Vázquez-Acevedo et al., The mitochondrial ATP synthase of chlorophycean algae contains eight subunits of unknown origin involved in the formation of an atypical stator-stalk and in the dimerization of the complex. J Bioenerg Biomembr. 38, 271-282 (2006).

5. R. van Lis, G. Mendoza-Hernández, G. Groth, A. Atteia, New insights into the unique structure of the FOF1-ATP synthase from the chlamydomonad algae Polytomella sp. and Chlamydomonas reinhardtii. Plant Physiol. 144, 1190-1199 (2007).

6. D. A. Cherepanov, A. Y. Mulkidjanian, W. Junge, Transient accumulation of elastic energy in proton translocating ATP synthase. FEBS Lett. 449, 1-6 (1999).

7. N. Soga, K. Kimura, K. Kinosita, M. Yoshida, T. Suzuki, Perfect chemomechanical coupling of FoF1-ATP synthase. Proc Natl Acad Sci U S A. 114, 4960-4965 (2017).

8. A. Wächter et al., Two rotary motors in F-ATP synthase are elastically coupled by a flexible rotor and a stiff stator stalk. Proc Natl Acad Sci U S A. 108, 3924-3929 (2011).

9. H. Sielaff et al., Domain compliance and elastic power transmission in rotary $F(O) F(1)-A T P a s e$. Proc Natl Acad Sci U S A. 105, 17760-17765 (2008).

10. J. L. Martin, R. Ishmukhametov, D. Spetzler, T. Hornung, W. D. Frasch, Elastic coupling power stroke mechanism of the F1-ATPase molecular motor. Proc Natl Acad Sci U S A. 115, 57505755 (2018). 
11. A. Zhou et al., Structure and conformational states of the bovine mitochondrial ATP synthase by cryo-EM. elife. 4, e10180 (2015).

12. M. Sobti et al., Cryo-EM structures of the autoinhibited E. coli ATP synthase in three rotational states. elife. 5 (2016), doi:10.7554/eLife.21598.

13. A. Hahn, J. Vonck, D. J. Mills, T. Meier, W. Kühlbrandt, Structure, mechanism, and regulation of the chloroplast ATP synthase. Science. 360 (2018), doi:10.1126/science.aat4318.

14. J. Zhao, S. Benlekbir, J. L. Rubinstein, Electron cryomicroscopy observation of rotational states in a eukaryotic V-ATPase. Nature. 521, 241-245 (2015).

15. Y. Komoriya et al., Principal role of the arginine finger in rotary catalysis of F1-ATPase. J Biol Chem. 287, 15134-15142 (2012).

16. M. D. Greene, W. D. Frasch, Interactions among gamma R268, gamma Q269, and the beta subunit catch loop of Escherichia coli F1-ATPase are important for catalytic activity. J Biol Chem. 278, 51594-51598 (2003).

17. K. Okazaki, G. Hummer, Elasticity, friction, and pathway of $\gamma$-subunit rotation in FoF1-ATP synthase. Proc Natl Acad Sci U S A. 112, 10720-10725 (2015).

18. S. Yanagisawa, W. D. Frasch, Protonation-dependent stepped rotation of the F-type ATP synthase c-ring observed by single-molecule measurements. J Biol Chem. 292, 17093-17100 (2017).

19. R. Ishmukhametov, T. Hornung, D. Spetzler, W. D. Frasch, Direct observation of stepped proteolipid ring rotation in E. coli FoF $F_{1}$ ATP synthase. EMBO J. 29, 3911-3923 (2010).

20. V. Giorgio et al., Cyclophilin D modulates mitochondrial F0F1-ATP synthase by interacting with the lateral stalk of the complex. J Biol Chem. 284, 33982-33988 (2009).

21. K. M. Johnson et al., Identification and validation of the mitochondrial F1F0-ATPase as the molecular target of the immunomodulatory benzodiazepine Bz-423. Chem Biol. 12, 485-496 (2005).

22. A. Vassilopoulos et al., SIRT3 deacetylates ATP synthase F1 complex proteins in response to nutrient- and exercise-induced stress. Antioxid Redox Signal. 21, 551-564 (2014).

23. J. Zheng, V. D. Ramirez, Purification and identification of an estrogen binding protein from rat brain: oligomycin sensitivity-conferring protein (OSCP), a subunit of mitochondrial FOF1-ATP synthase/ATPase. J Steroid Biochem Mol Biol. 68, 65-75 (1999).

24. J. Zheng, V. D. Ramirez, Rapid inhibition of rat brain mitochondrial proton FOF1-ATPase activity by estrogens: comparison with $\mathrm{Na+}, \mathrm{K}+$-ATPase of porcine cortex. Eur J Pharmacol. 368, 95102 (1999).

25. F. Massart, S. Paolini, E. Piscitelli, M. L. Brandi, G. Solaini, Dose-dependent inhibition of mitochondrial ATP synthase by 17 $\beta$-estradiol. Gynecol Endocrinol. 16, 373-377 (2002). 
26. J. W. Simpkins, K. D. Yi, S.-H. Yang, J. A. Dykens, Mitochondrial mechanisms of estrogen neuroprotection. Biochim Biophys Acta. 1800, 1113-1120 (2010).

27. M. Bergeaud et al., Mitochondrial p53 mediates a transcription-independent regulation of cell respiration and interacts with the mitochondrial $F_{1}$ FO-ATP synthase. Cell Cycle. 12, 2781-2793 (2013).

28. P. Balabaskaran Nina et al., ATP synthase complex of Plasmodium falciparum: dimeric assembly in mitochondrial membranes and resistance to genetic disruption. J Biol Chem. 286, 41312-41322 (2011).

29. E. Perez et al., The mitochondrial respiratory chain of the secondary green alga Euglena gracilis shares many additional subunits with parasitic Trypanosomatidae. Mitochondrion. 19 Pt B, 338-349 (2014).

30. I. Starke, G. D. Glick, M. Börsch, Visualizing Mitochondrial FoF1-ATP Synthase as the Target of the Immunomodulatory Drug Bz-423. Front Physiol. 9, 803 (2018).

31. B. Rieger, W. Junge, K. B. Busch, Lateral pH gradient between OXPHOS complex IV and $F(0) F(1)$ ATP-synthase in folded mitochondrial membranes. Nat Commun. 5, 3103 (2014).

32. D. Pogoryelov et al., Microscopic rotary mechanism of ion translocation in the $F(0)$ complex of ATP synthases. Nat Chem Biol. 6, 891-899 (2010).

33. B. D. Cain, R. D. Simoni, Interaction between Glu-219 and His-245 within the a subunit of F1F0ATPase in Escherichia coli. J Biol Chem. 263, 6606-6612 (1988).

34. V. Leone, D. Pogoryelov, T. Meier, J. D. Faraldo-Gómez, On the principle of ion selectivity in $\mathrm{Na}+/ \mathrm{H}+$-coupled membrane proteins: experimental and theoretical studies of an ATP synthase rotor. Proc Natl Acad Sci U S A. 112, E1057-66 (2015).

35. H. Guo, S. A. Bueler, J. L. Rubinstein, Atomic model for the dimeric Fo region of mitochondrial ATP synthase. Science. 358, 936-940 (2017).

36. E. Lewis, C. Munger, R. Watson, D. Wise, Life Cycle of Polytomella caeca (Phytomonadida, Polyblepharidae). J Protozool. 21, 647-649 (1974).

37. J. A. Vizcaíno et al., 2016 update of the PRIDE database and its related tools. Nucleic Acids Res. 44, D447-56 (2016).

38. E. Zerbetto, L. Vergani, F. Dabbeni-Sala, Quantification of muscle mitochondrial oxidative phosphorylation enzymes via histochemical staining of blue native polyacrylamide gels. Electrophoresis. 18, 2059-2064 (1997).

39. I. Rais, M. Karas, H. Schägger, Two-dimensional electrophoresis for the isolation of integral membrane proteins and mass spectrometric identification. Proteomics. 4, 2567-2571 (2004).

40. A. Punjani, J. L. Rubinstein, D. J. Fleet, M. A. Brubaker, cryoSPARC: algorithms for rapid unsupervised cryo-EM structure determination. Nat Methods. 14, 290-296 (2017). 
41. P. V. Afonine et al., New tools for the analysis and validation of cryo-EM maps and atomic models. Acta crystallographica. Section D, Structural biology. 74, 814-840 (2018).

42. P. Emsley, B. Lohkamp, W. G. Scott, K. Cowtan, Features and development of Coot. Acta Crystallogr D Biol Crystallogr. 66, 486-501 (2010).

43. N. Eswar et al., Tools for comparative protein structure modeling and analysis. Nucleic Acids Res. 31, 3375-3380 (2003).

44. E. Morales-Rios, M. G. Montgomery, A. G. W. Leslie, J. E. Walker, Structure of ATP synthase from Paracoccus denitrificans determined by $\mathrm{X}$-ray crystallography at $4.0 \AA$ A resolution. Proc Natl Acad Sci U S A. 112, 13231-13236 (2015).

45. J. R. Gledhill, M. G. Montgomery, A. G. W. Leslie, J. E. Walker, How the regulatory protein, IF(1), inhibits F(1)-ATPase from bovine mitochondria. Proc Natl Acad Sci U S A. 104, 1567115676 (2007).

46. G. C. Robinson et al., The structure of $F_{1}$-ATPase from Saccharomyces cerevisiae inhibited by its regulatory protein $\mathrm{IF}_{1}$. Open Biol. 3, 120164 (2013).

47. I. N. Watt, M. G. Montgomery, M. J. Runswick, A. G. W. Leslie, J. E. Walker, Bioenergetic cost of making an adenosine triphosphate molecule in animal mitochondria. Proc Natl Acad Sci U SA. 107, 16823-16827 (2010).

48. P. V. Afonine et al., Real-space refinement in PHENIX for cryo-EM and crystallography. Acta crystallographica. Section D, Structural biology. 74, 531-544 (2018).

49. E. F. Pettersen et al., UCSF Chimera-a visualization system for exploratory research and analysis. J Comput Chem. 25, 1605-1612 (2004).

50. T. D. Goddard et al., UCSF ChimeraX: Meeting modern challenges in visualization and analysis. Protein Sci. 27, 14-25 (2018).

51. J. Fan, R. W. Lee, Mitochondrial genome of the colorless green alga Polytomella parva: two linear DNA molecules with homologous inverted repeat Termini. Mol Biol Evol. 19, 999-1007 (2002).

52. S. Buschmann et al., The structure of $\mathrm{cbb3}$ cytochrome oxidase provides insights into proton pumping. Science. 329, 327-330 (2010).

53. G. M. Boratyn et al., Domain enhanced lookup time accelerated BLAST. Biol Direct. 7, 12 (2012).

54. D. M. Goodstein et al., Phytozome: a comparative platform for green plant genomics. Nucleic Acids Res. 40, D1178-86 (2012).

55. F. Sievers, D. G. Higgins, Clustal Omega for making accurate alignments of many protein sequences. Protein Sci. 27, 135-145 (2018). 
56. A. M. Waterhouse, J. B. Procter, D. M. A. Martin, M. Clamp, G. J. Barton, Jalview Version 2--a multiple sequence alignment editor and analysis workbench. Bioinformatics. 25, 1189-1191 (2009).

57. S. Q. Zheng et al., MotionCor2: anisotropic correction of beam-induced motion for improved cryo-electron microscopy. Nat Methods. 14, 331-332 (2017).

58. A. Rohou, N. Grigorieff, CTFFIND4: Fast and accurate defocus estimation from electron micrographs. J Struct Biol. 192, 216-221 (2015).

59. J. Zivanov et al., New tools for automated high-resolution cryo-EM structure determination in RELION-3. elife. 7 (2018), doi:10.7554/eLife.42166.

60. M. Vázquez-Acevedo et al., Dissecting the peripheral stalk of the mitochondrial ATP synthase of chlorophycean algae. Biochim Biophys Acta. 1857, 1183-1190 (2016).

61. A. Cano-Estrada et al., Subunit-subunit interactions and overall topology of the dimeric mitochondrial ATP synthase of Polytomella sp. Biochim Biophys Acta. 1797, 1439-1448 (2010).

Acknowledgements: This work was funded by the Max Planck Society. BJM acknowledges funding from an EMBO Long-term fellowship (EMBO LTF 702-2016). Author contributions: WK initiated and supervised the study. NK grew Polytomella cultures, isolated, purified and analysed ATP synthase dimers biochemically. BJM and NK prepared cryoEM specimens and collected data. BJM performed image analysis and identified and analysed rotary substates. JL performed mass spectrometry. DJM maintained cryoEM instrumentation and EM alignment. BJM, NK and JL, ÖY, WK analysed data. BJM, NK and WK wrote the manuscript. Competing Interests: Authors declare no competing interests.

Data and materials availability: Maps and models are publicly available through the EMDB and PDB databases (Tables S2 and S3). Mass spectrometry data are publicly available through the PRIDE database (accession number XXXX)(37).

\section{Supplemental Materials:}

Materials and Methods

Figures S1-S9

Tables S1-S3

Movies S1-S5

References 37-61 

available under aCC-BY-NC 4.0 International license.

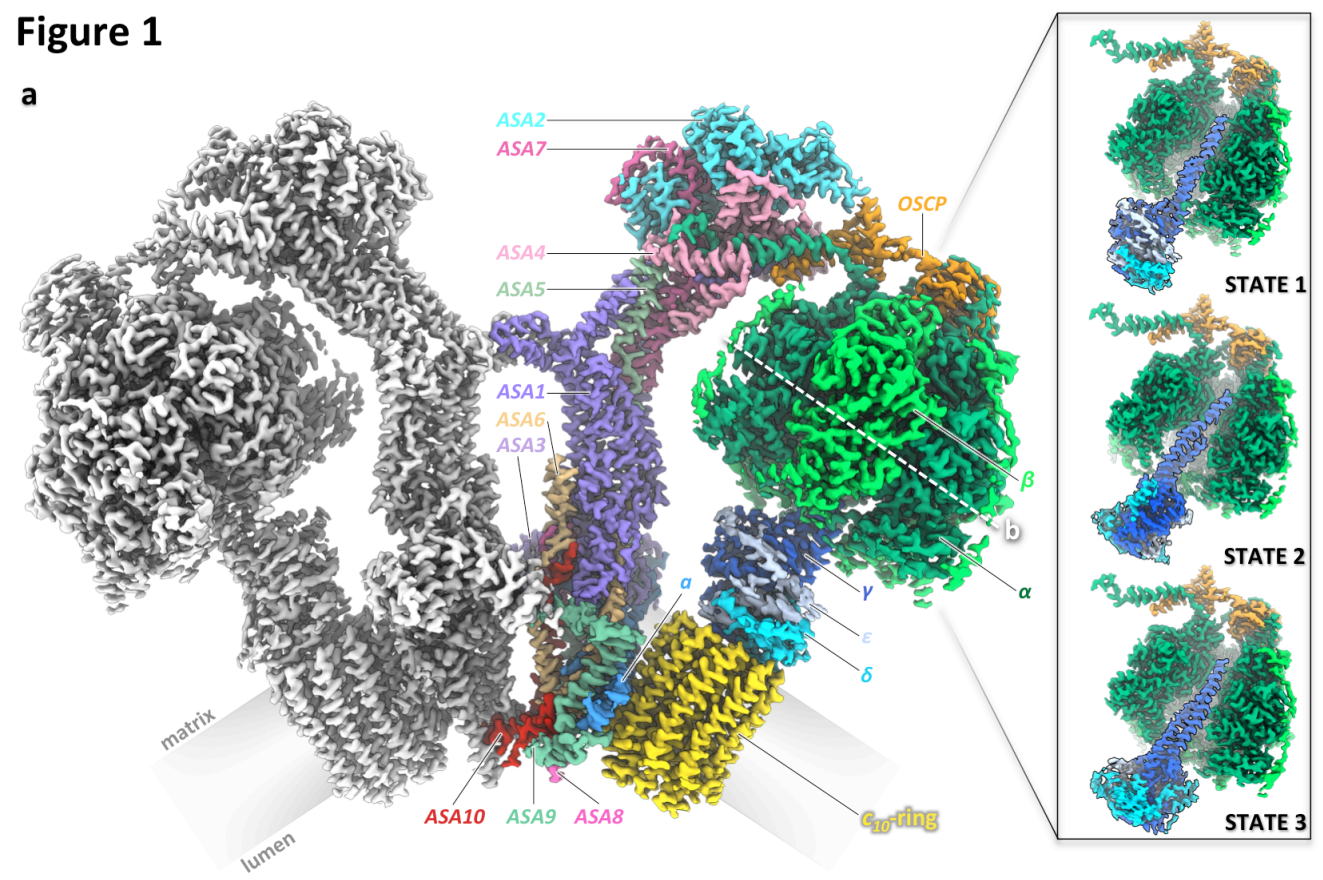

b

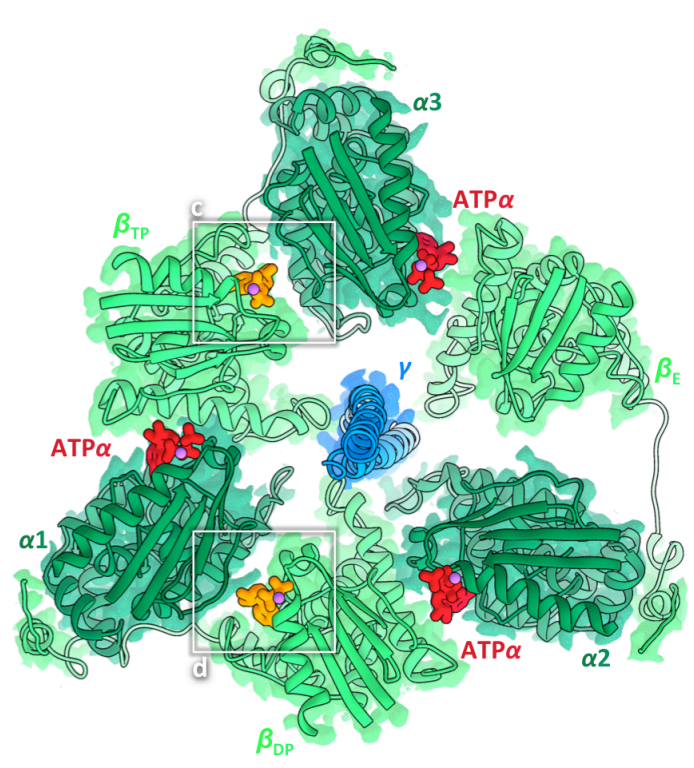

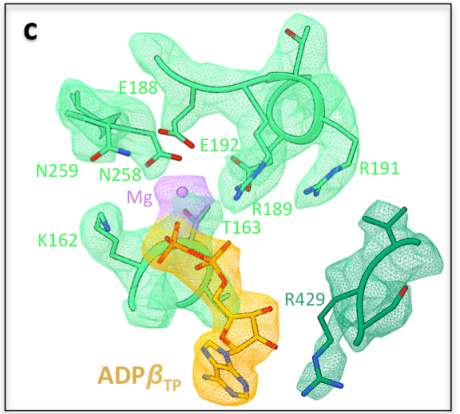

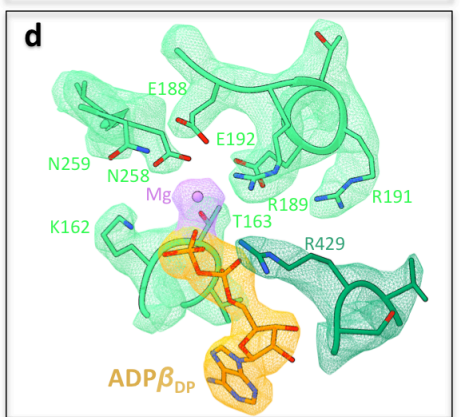

Figure 1. High-resolution structure of the mitochondrial $F_{1} F_{o}$ ATP synthase dimer from Polytomella sp. a)

Composite cryo-EM map density of the 62-subunit, 1.58 MDa Polytomella dimer, colored by subunit on the right. The $c_{10}$ rotor ring (yellow) and subunit $a$ (bright blue) make up the $\mathrm{F}_{\mathrm{o}}$ motor complex. Proton translocation through $\mathrm{F}_{\mathrm{o}}$ causes rotation of the $c$-ring and the attached central stalk subunits $\gamma$ (dark blue), $\delta$ (cyan) and $\varepsilon$ (light blue). The $\mathrm{F}_{1}$ head consists of three catalytic $\beta$ subunits (bright green) and three $\alpha$ subunits (dark green). Long C-terminal extensions of $\beta$ wrap around the $\alpha$ subunits on the outside of $\mathrm{F}_{1}$ (see Movie S5). The two-domain OSCP subunit (orange) links the three $\alpha$ subunits to the peripheral stalk subunits ASA1-10 (see Figure 4). Subunit $A S A 10$ (red) connects the two $F_{1} F_{0}$ monomers in the membrane (see Figure S7, S9). The local map resolution is $2.7 \AA$ for the peripheral stalk, $F_{o}$ and $c$-ring, and 2.8 to $2.9 \AA$ for the $F_{1}$ head and central stalk (see Figure S2). Inset: Three primary rotary states 1,2 and 3 of the $\mathrm{F}_{1} \mathrm{~F}_{\mathrm{o}}$ monomer are related by $\sim 120^{\circ}$ rotation of the central stalk within the $\alpha_{3} \beta_{3}$ assembly. Unless otherwise specified, subunit coloring is consistent throughout all figures. b) Section through $\mathrm{F}_{1}$ at the level of bound nucleotides. $\beta_{\mathrm{DP}}$ and $\beta_{\mathrm{TP}}$ sites contain ADP (orange) and $\beta_{\mathrm{E}}$ is empty. The three $\alpha$-subunits bind a structural ATP (red). Nucleotide-coordinating $\mathrm{Mg}^{2+}$ ions are violet. The central stalk subunit $\gamma$ engages with the catch loop of the $\beta_{\mathrm{E}}$ subunit (see Figure S5). c,d) Catalytic sites of subunits $\beta_{\mathrm{DP}}$ and $\beta_{\mathrm{TP}}$. A well-defined arginine sidechain of subunit $\alpha$ (the "arginine finger") extends toward the nucleotide phosphate in the $\beta_{\mathrm{DP}}$ site. ATP in the $\beta_{\text {TP }}$ site has hydrolyzed to ADP during protein isolation. 


\section{Figure 2}

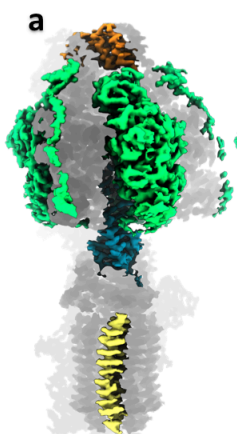

$1 \mathrm{~A}$

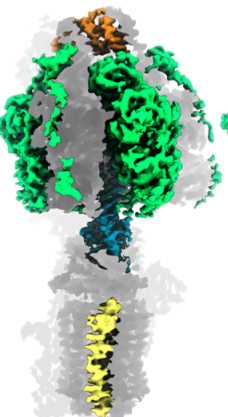

1B

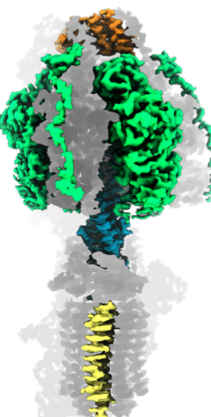

$1 \mathrm{C}$

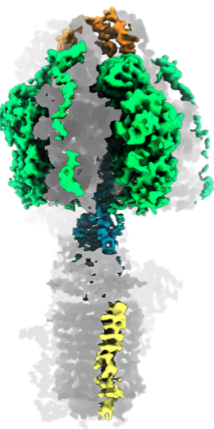

1D

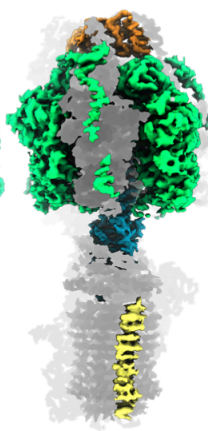

$1 \mathrm{E}$

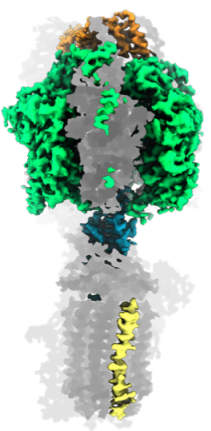

1F

b
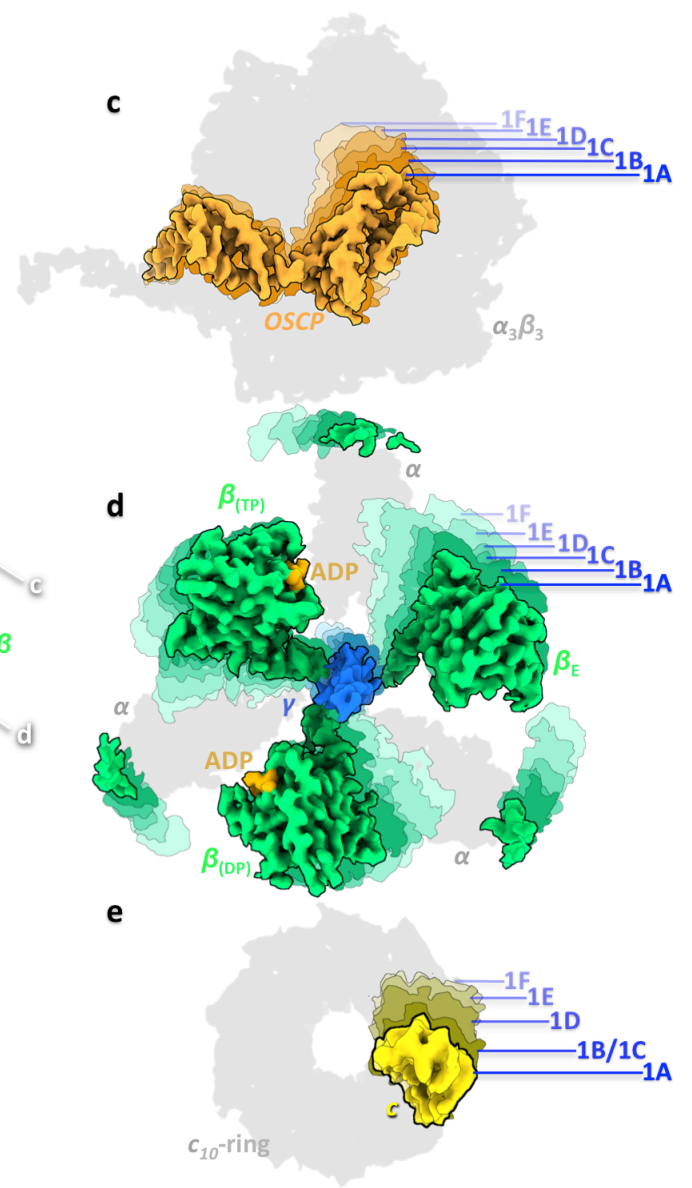

Figure 2. Rotary substates of mitochondrial ATP synthase. The three primary rotary states are subdivided into a total of 13 rotary substates at resolutions between 2.8 and $4.2 \AA$ (see Figure S1 and Table S2). The number of resolved substates and angular increments between them differ for each of the three primary rotary states. a) Density maps of rotary substates $1 \mathrm{~A}$ to $1 \mathrm{~F}$ ordered in ATP synthesis direction according to the position of the central stalk with respect to the peripheral stalk. b) Composite cryo-EM density map of rotary substate $1 \mathrm{~A}$ at $2.9 \AA$ resolution. c-e) An overlay of substate maps indicates a concerted movement of OSCP (orange, c), the $F_{1}$ head (green, d), $\gamma$ (blue, d), and the $c$-ring (yellow, e) from substate 1A to 1F. Projected map densities of other subunits are shown in light grey for reference. 


\section{Figure 3}

STATE 1

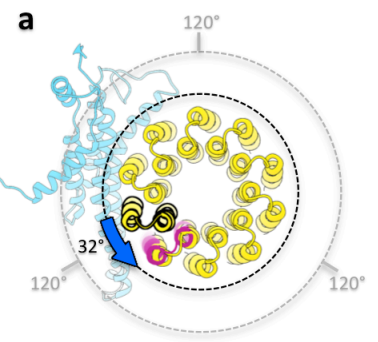

b

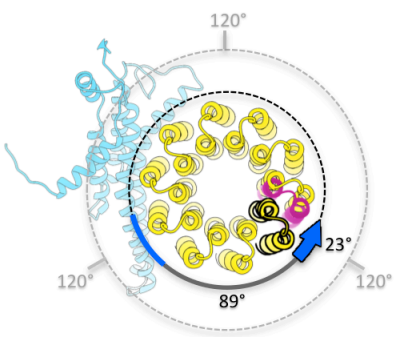

STATE 2
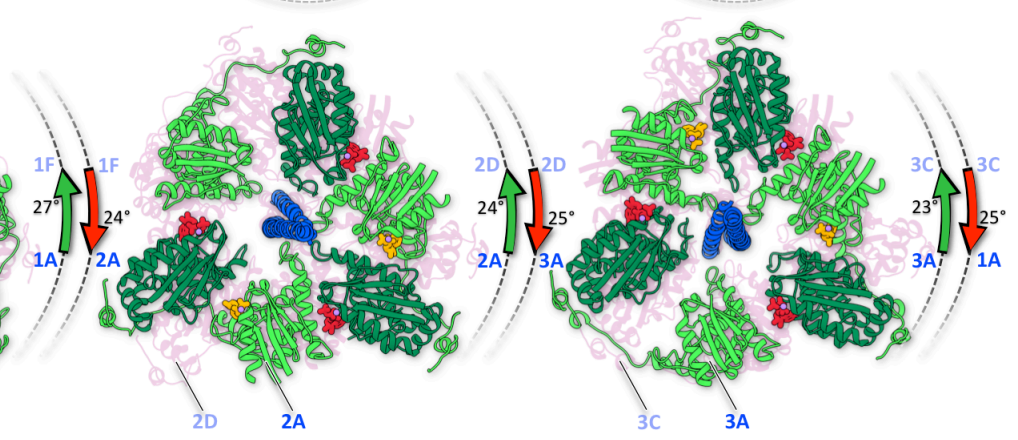

STATE 3
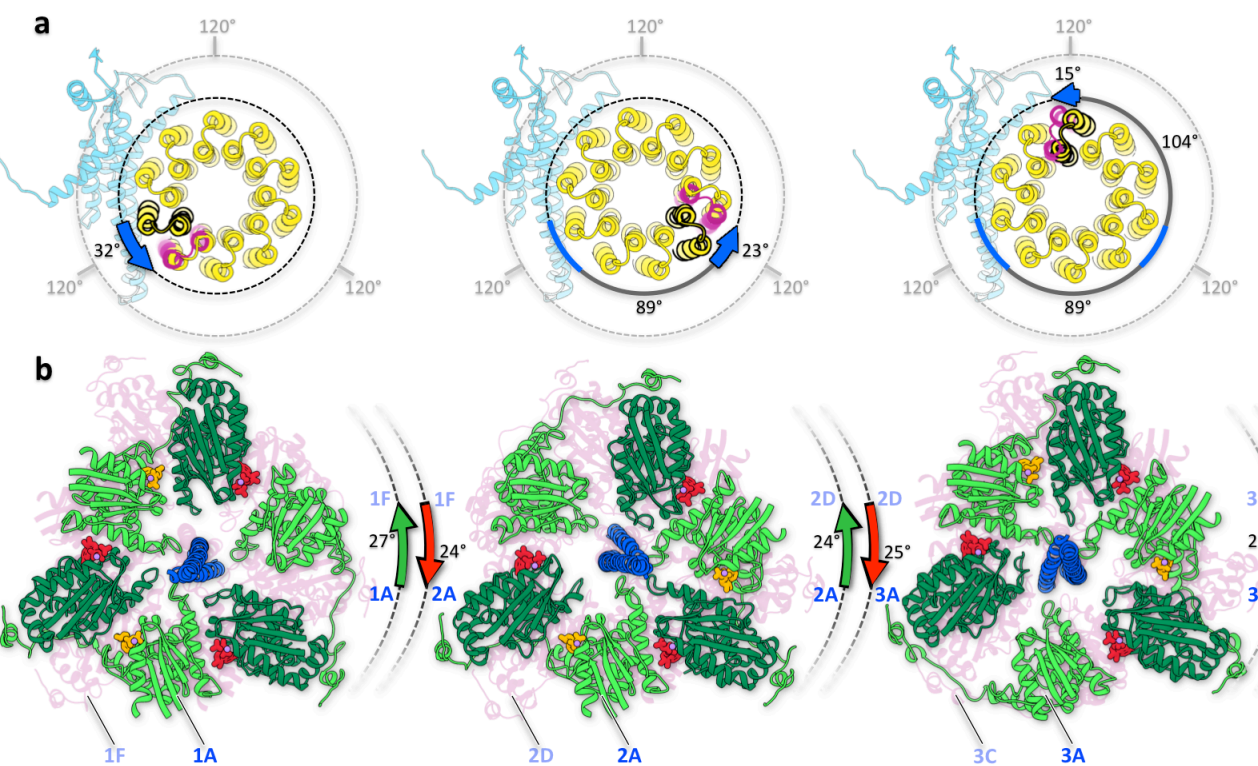

Figure 3. Concerted rotation of $\mathbf{F}_{1}$ with central stalk and $\boldsymbol{c}$-ring. a) Progressing in ATP synthesis direction, the $c_{10}$-ring rotates counter-clockwise (as seen from $\mathrm{F}_{1}$ ) with respect to the $a$-subunit, by up to $32^{\circ}$ for substates of the same primary rotary state. The primary rotary states differ by power strokes of $\sim 120^{\circ}$. b) Between substates of a given rotary state, the $F_{1}$ head rotates together with the $c$-ring and central stalk before recoiling to its original position in the first substate of the subsequent primary rotary state. 


\section{Figure 4}

a
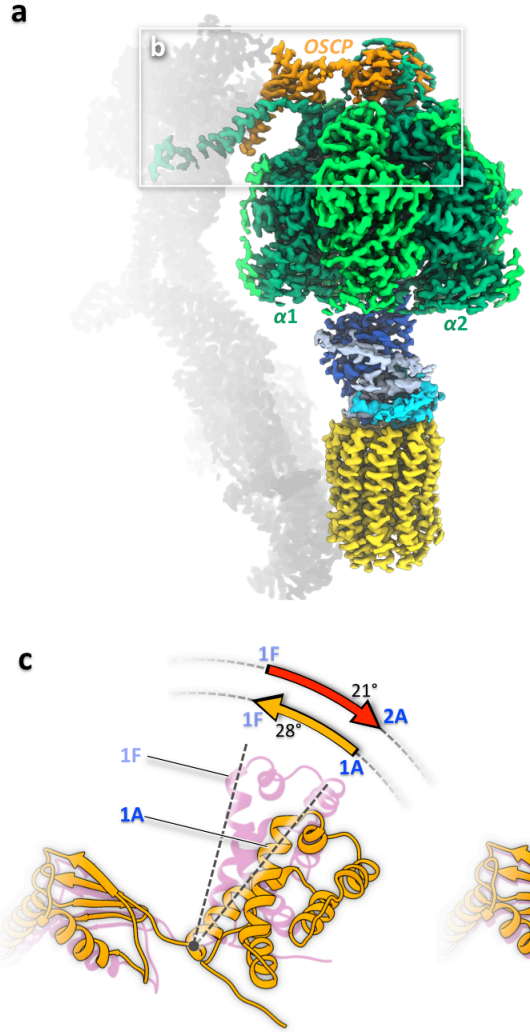

STATE 1

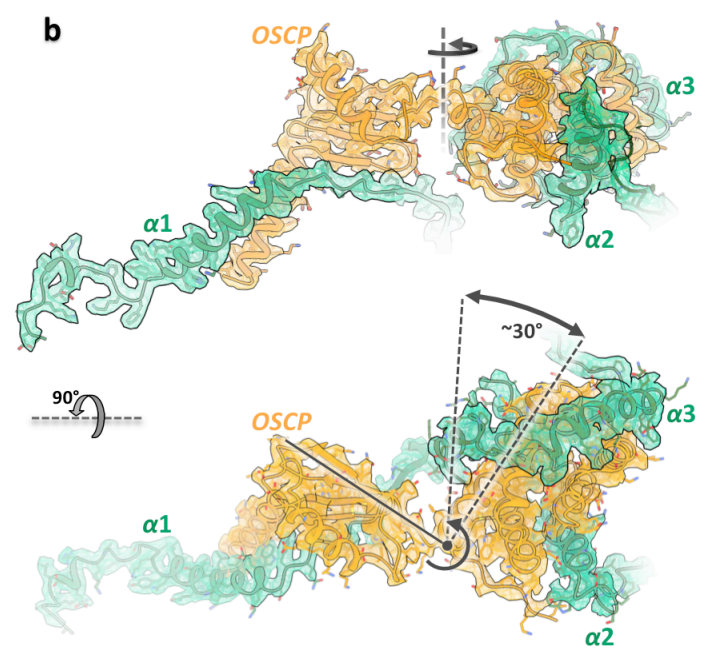

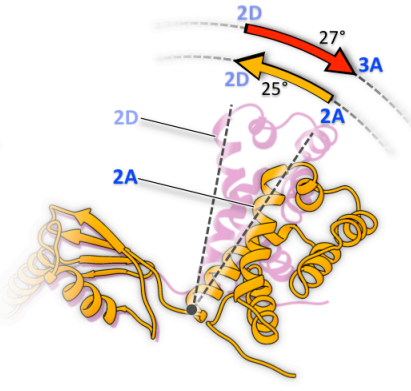

STATE 2

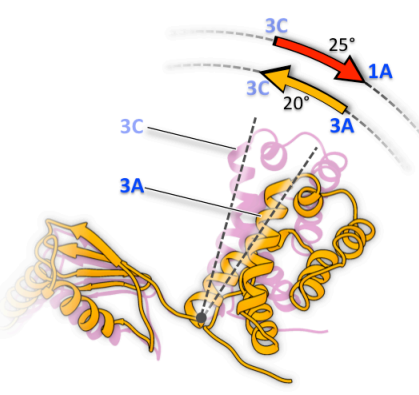

STATE 3

Figure 4. Subunit $O S C P$ connects the $F_{1}$ head and peripheral stalk as a flexible hinge. a) Overview of $F_{1} F_{o}$ monomer indicating the position of subunit OSCP (orange) on top of the $F_{1}$ head (green) relative to the peripheral stalk (gray). b) The two-domain OSCP interacts with the helical C-terminal extensions of subunits $\alpha 1, \alpha 2$ and $\alpha 3$ (green) (see Movie S4). Extensions of $\alpha 2$ and $\alpha 3$ form short helix bundles with helices of the globular $\mathrm{N}$-terminal OSCP domain, while $\alpha 1$ binds to the elongated C-terminal OSCP domain that is attached to the peripheral stalk via the $\alpha 1$ extension. The two OSCP domains are connected by a flexible peptide link that facilitates $\sim 30^{\circ}$ back and forth rotation of the $\mathrm{N}$-terminal domain with $\mathrm{F}_{1}$ (black arrows) relative to the $\mathrm{C}$-terminal $\mathrm{OSCP}$ domain and peripheral stalk. c) Between substates of a given primary rotary state, the $\mathrm{N}$-terminal OSCP domain rotates with $\mathrm{F}_{1}$ by up to $28^{\circ}$ (straight dashed lines) in synthesis direction (orange arrows) and then recoils to its starting position in the first substate of the next primary rotary state (red arrows). 


\section{Figure 5}

a

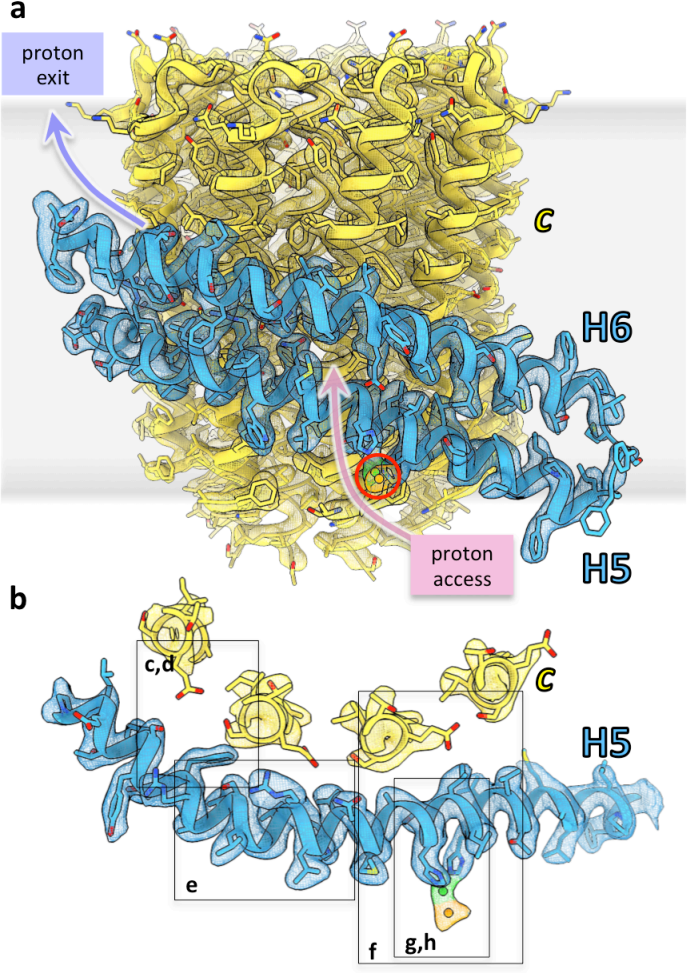

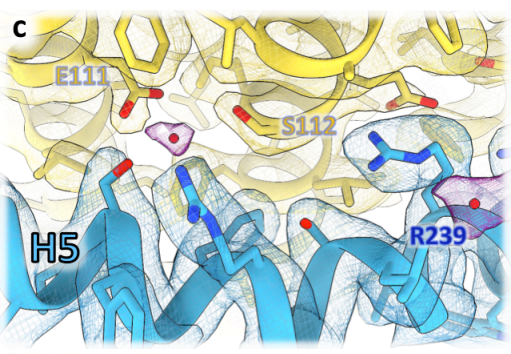
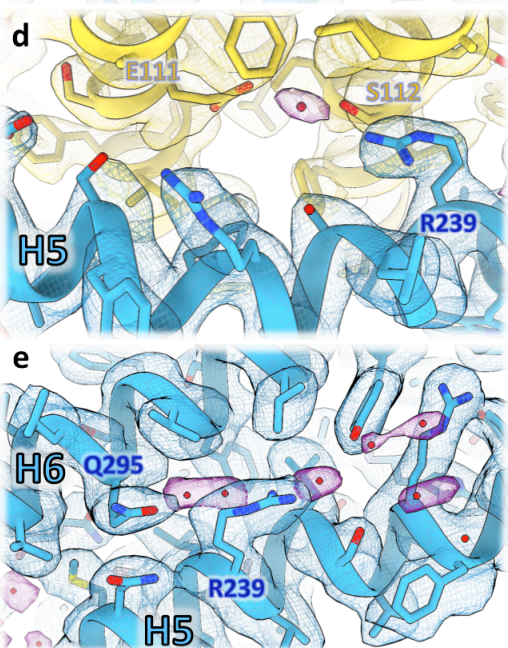

h c-ring position 2

$\mathbf{f}$
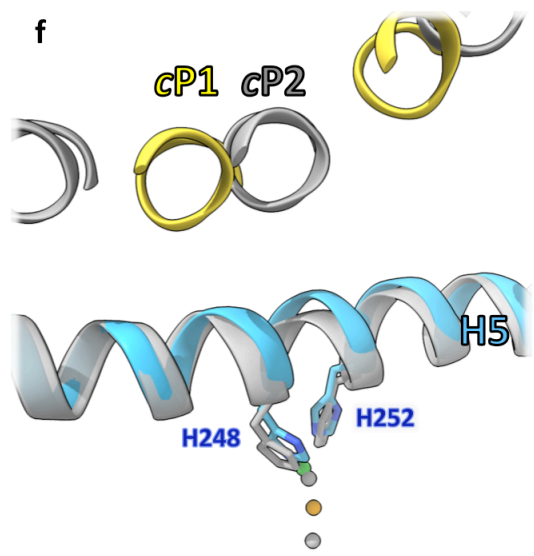

g c-ring position 1
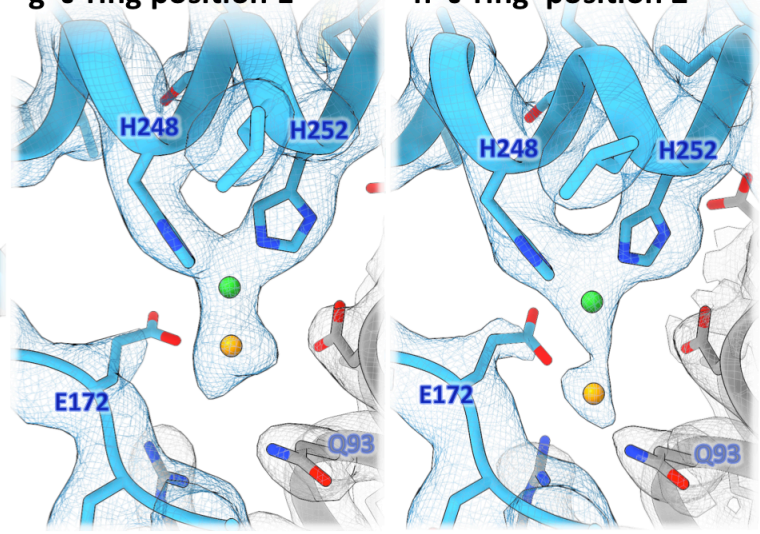

Figure 5. Metal ion and ordered water molecules in the $\mathrm{F}_{\mathrm{o}}$ proton access and release channels. a) The H5/H6 helix hairpin of subunit $a$ (light blue) forms the bearing against which the $c_{10}$-ring (yellow) rotates, shown here for $c$-ring position 1. b) Sectional view of $\mathrm{H} 5$ and outer-ring $c$-subunit helices with proton-binding cGlu111, c-ring position 1. c,d) Water molecules (purple density) coordinated by the ion-binding cGlu111 and adjacent cSer112 in the matrix channel for c-ring positions 1 (c) and 2 (d). Maps are displayed at density thresholds of 0.035 for subunit $a, 0.025$ for the $c$-ring, and 0.025 (c) or 0.018 (d) for water. e) The strictly conserved $a$ Arg239 and $a$ Gln295 that separate the proton access and release channels in the membrane bind two water molecules, shown in the concensus $\mathrm{C2}$-refined map of the $\mathrm{F}_{\mathrm{o}}$ region. $\mathbf{f}-\mathbf{h}$ ) Conserved residues $\mathrm{aHis} 248$ and $a \mathrm{His} 252$ in $\mathrm{H} 5$ of subunit $a$ coordinate a metal ion. Bond distances change depending on c-ring position. The predominant $c$-ring position (position 1 , colored, and panel $\mathbf{h}$ ) accounts for $58 \%$ of particles, while a second position, differing by rotation through roughly $13^{\circ}$, (position 2 , gray, and panel $\mathbf{g}$ ) accounts for $33 \%$. The overlay of $\mathbf{g}$ and $\mathbf{h}$ in panel $\mathbf{f}$ indicates a small lateral displacement of $\mathbf{H} 5$ with its metal-coordinating sidechains. Except where specified, all maps in a given panel are rendered at the same density threshold. 
bioRxiv preprint doi: https://doi org/10.1101/543108: this version posted February 6, 2019. The copyright holder for this preprint (which was not certified by peer review) is the author/funder, who has granted bioRxiv a license to display the preprint in perpetuity. It is made available under aCC-BY-NC 4.0 International license.

\section{Figure 6}

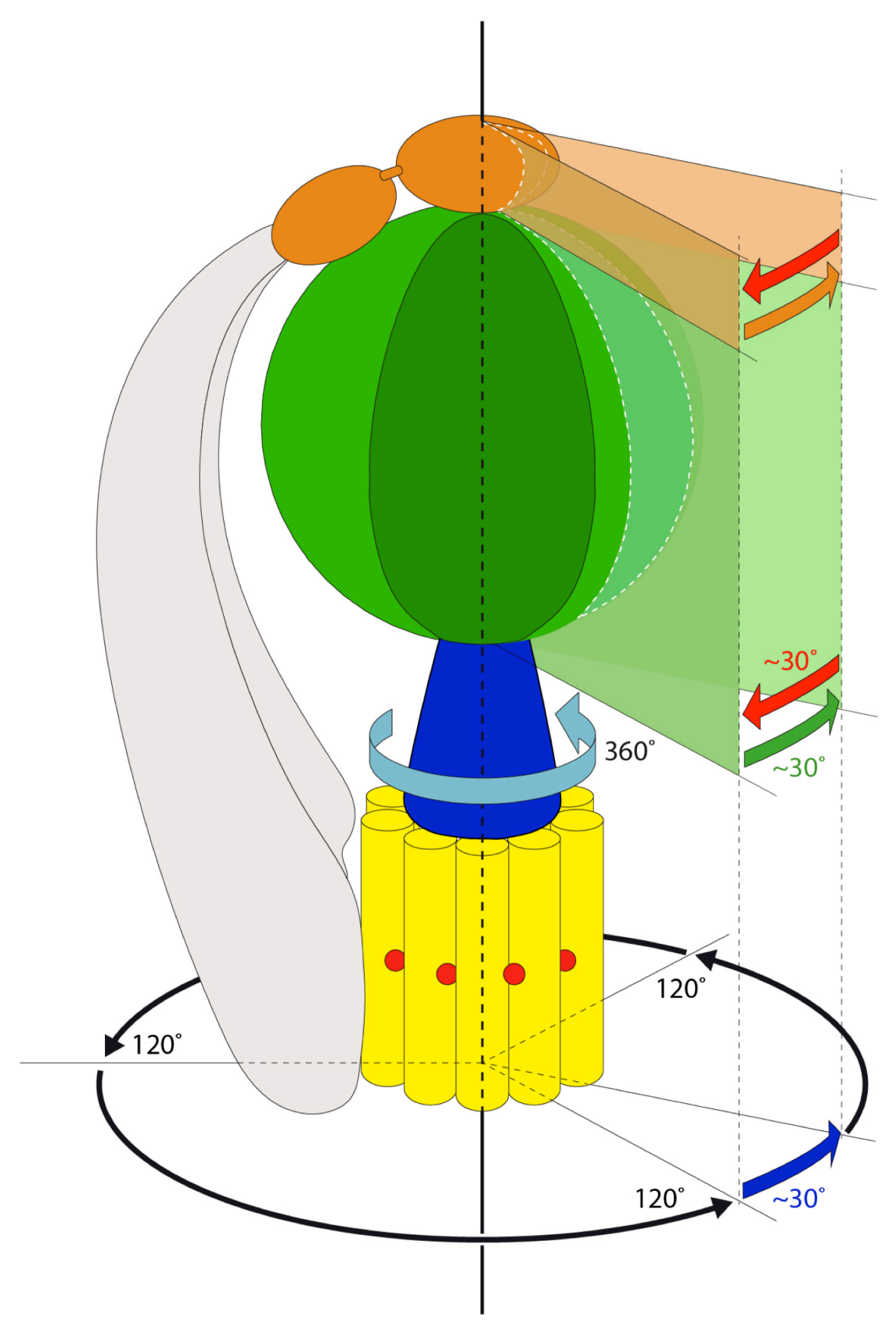

Figure 6. Concerted movement of $F_{1}$ and rotor in $F_{1} F_{0}$ ATP synthase facilitates flexible coupling of $F_{0}$ and $F_{1}$. The $F_{1}$ head (green) rotates together with the $c$-ring rotor (yellow) and central stalk (blue) for the first $20-30^{\circ}$ of each $\sim 120^{\circ}$ primary rotary step. OSCP (orange) forms a flexible link between the $F_{1}$ head and the peripheral stalk (gray). $F_{1}$ and the proximal OSCP domain then together recoil to their original position and the central stalk rotates $\sim 120^{\circ}$ within $\mathrm{F}_{1}$ to the next primary rotary state. In this way, rotation of the central stalk within $\alpha_{3} \beta_{3}$ and rotation of the $c_{10}$-ring in $\mathrm{F}_{\mathrm{o}}$ are flexibly coupled. 